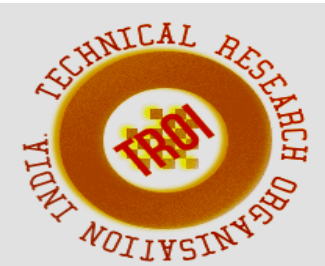

\title{
AN AUTOMATIC DROWSINESS AND ALCOHOL DETECTION SYSTEM USING IOT

\author{
Shalini $\mathrm{G}^{1}$, Monisha Aishwaraya R A ${ }^{2}$, Madhusha $\mathrm{M}^{3}$, Chitra $\mathrm{S}^{4}$, Andrea Serena $\mathrm{J}^{5}$ \\ 1,2,3,4,5DR.TTIT KGF, \\ shalini.reddy.leo@gmail.com ${ }^{1}$, monishaaishwaraya@gmail.com ${ }^{2}, \underline{\text { madhusha3005@gmail.com }}^{3}$ \\ chitrasn555@gmail.com ${ }^{4}$
}

\begin{abstract}
Drowsy is the reason for most of the road accidents. Manually tracing the drowsy driver is not an easy task. So a system that must be developed in every car and it detects the drowsy driver, detects the alcohol consumption of the driver and also detects whether the person uses mobile while driving. Driver fatigue, alcohol consumption and using mobile while driving often becomes a direct cause of many traffic accidents. Therefore, there is a need to develop the systems that will detect and caution a driver of her/him condition, which could significantly reduce the number of car accidents caused related to these aspects . However, the development of such systems encounters many difficulties related to fast and proper recognition of a driver's symptoms.

Index Terms: Atmel, Buffer, Driver, LCD, Eye Blink sensor, Mobile Sensor, MQ3 sensor, Relay, RF Transmitter and Receiver, Power Supply.
\end{abstract}

\section{I.INTRODUCTION}

Driver drowsiness detection is a car safety technology which helps prevent accidents caused by the driver getting drowsy. Various studies have suggested that around $20 \%$ of all road accidents are fatigue-related, up to $50 \%$ on certain roads. Some of the current systems learn driver patterns and can detect when a driver is becoming drowsy. And the accidens is also caused due to alcohol consumption and when the driver is speaking in the phone while driving. The development of technologies for detecting or preventing drowsiness, alcohol consumption detection and mobile phone detection at the wheel is a major challenge in the field of accident avoidance systems. Because of the hazard that presents on the road, methods need to be developed for counteracting its affects [2].

The aim of this project is to develop a prototype drowsiness detection, alcohol consumption detection and mobile phone detection system. The focus will be placed on designing a system that will accurately monitor the eye blink rate, alcohol consumption level and mobile phone usage. In this project we use sensors to measure all these factors. The values measured will be sent to the microcontroller where the measured values will be compared with the reference values. If the values measured do not match with the reference values then the microcontroller will send a warning signal in the LCD display thereby preventing accidents.

\section{PROPOSED ALERT UNIT SYSTEM}

This is a small system, so we can easily embed it on any vehicle. The Eye blink sensor is fixed to the driver. The eye blink sensor senses the movement of the eyeball. The sensor output is connected to a aurdino. The car engine starting system is directly controlled by the aurdino. If the sensor detects the no output from the sensor because there no movement in the eyeball, it sends the signal to the aurdino. The aurdino immediately buzzes the buzzer, also give warning signal and display the reason in a $\mathrm{LCD}[5]$.

The system is developed by interfacing a alcohol sensor, eye blink sensor and a mobile sensor with an ADC which converts the analog 
readings to digital, thus extracted digital data is processed using a aurdino[5]. The reference values of these three parameters aurdino memory[1]. If anyone of these three parameter exceeds the reference value the microcontroller automatically performs the action specified to do for that particular input received from that particular sensor. The microcontroller used here is AMTEL89X51, it has an inbuilt ADC and counters, and the counter is used to count heartbeat, respirator rate and ADC for converting analog temperature to digital.

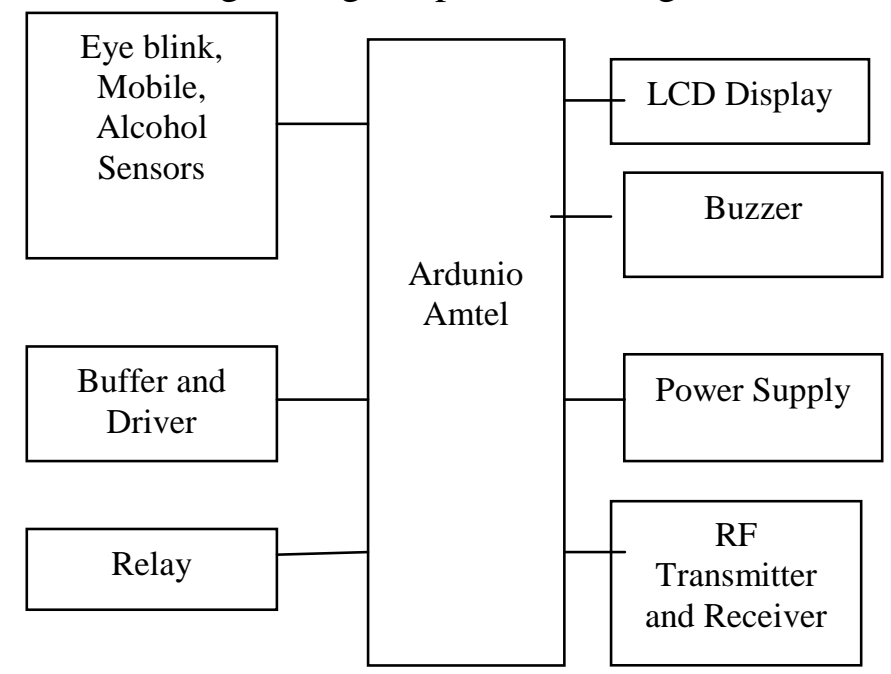

Figure 1:Block diagram of the proposed system

\section{Description of the Block Diagram Atmel 89C51}

AT89C51 is an 8-bit microcontroller and belongs to Atmel's 8051 family. ATMEL 89C51 has $4 \mathrm{~KB}$ of Flash programmable and erasable read only memory (PEROM) and 128 bytes of RAM. It can be erased and program to a maximum of 1000 times.

\section{Atmel 89C51}

In 40 pin AT89C51, there are four ports designated as $\mathrm{P}_{1}, \mathrm{P}_{2}, \mathrm{P}_{3}$ and $\mathrm{P}_{0}$. All these ports are 8-bit bi-directional ports, i.e., they can be used as both input and output ports. Except $\mathrm{P}_{0}$ which needs external pull-ups, rest of the ports have internal pull-ups. When $1 \mathrm{~s}$ are written to these port pins, they are pulled high by the internal pull-ups and can be used as inputs. These ports are also bit addressable and so their bits can also be accessed individually. Port $\mathrm{P}_{0}$ and $\mathrm{P}_{2}$ are also used to provide low byte and high byte addresses, respectively, when connected to an external memory. Port 3 has multiplexed pins for special functions like serial communication, hardware interrupts, timer inputs and read/write operation from external memory. AT89C51 has an inbuilt UART for serial communication. It can be programmed to operate at different baud rates. Including two timers\& hardware interrupts, it has a total of six interrupts[3].

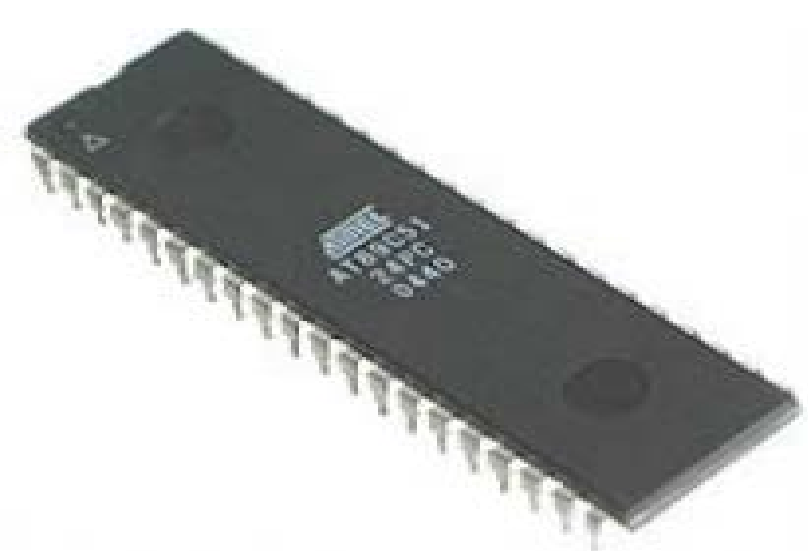

Figure.2 Atmel 89C51

\section{Power supply unit}

This section needs two voltages viz., $+12 \mathrm{~V} \&+5 \mathrm{~V}$, as working voltages. Hence specially designed power supply is constructed to get regulated power supplies as in figure 3 .

The circuit needs two different voltages, $+5 \mathrm{~V} \&$ $+12 \mathrm{~V}$, to work. These dual voltages are supplied by this specially designed power supply. The power supply, unsung hero of every electronic circuit, plays very important role in smooth running of the connected circuit. The main object of this 'power supply' is, as the name itself implies, to deliver the required amount of stabilized and pure power to the circuit[3]. The regulated supply as given below

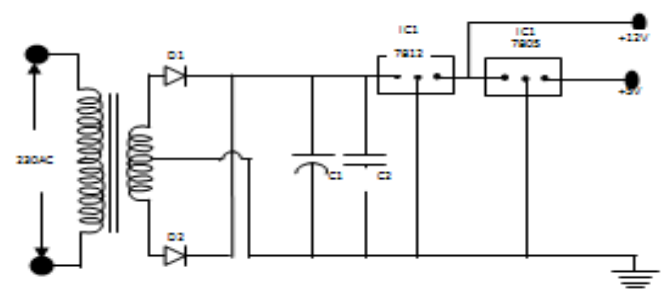

Figureure.3 Power Supply Circuit Diagram

\section{Circuit Description of Power Suppky}

A D.C. power supply which maintains the output voltage constant irrespective of A.C. mains fluctuations or load variations is known as regulated D.C. power supply. It is also referred as full-wave regulated power supply as it uses four diodes in bridge fashion with the 
transformer. This laboratory power supply offers excellent line and load regulation and output voltages of $+5 \mathrm{~V} \&+12 \mathrm{~V}$ at output currents up to one amp.

\section{Eye blink Sensor}

The Eye blink sensor works by illuminating the eye or eyelid area with infrared light, then monitoring the hanges in the reflected light using a photo transister and differentiator circuit. The exact functionality depends greatly on the positioning and aiming of the emitter and detector with respect to the eye[4].

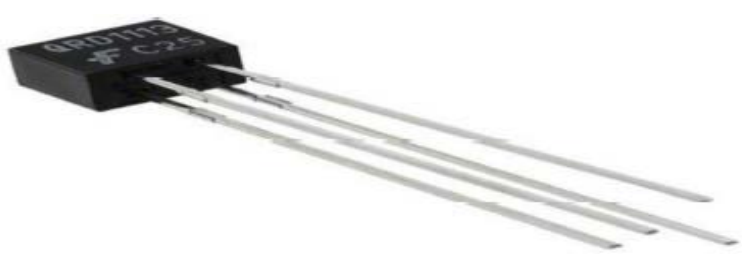

Figure.4 Eye Blink Sensor

\section{Alcohol Sensor}

MQ3 sensor is used for alcohol dectection.It is suitable for detecting Alcohol, Benzine, $\mathrm{CH} 4$, Hexane, LPG, CO. Due to its high sensitivity and fast response time, measurements can be taken as soon as possible. The sensitivity of the sensor can be adjusted by using the potentiometer[7].

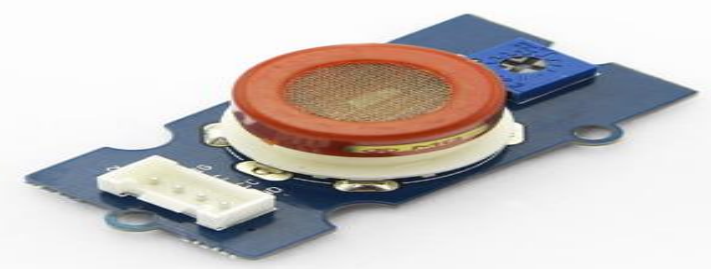

Figureure.5 Alcohol Sensor

\section{Mobile Sniffer}

This handy mobile bug or cell phone detector, pocket-size mobile transmission detector or sniffer can sense the presence of an activated mobile cellphone from a distance of one and-a-half meters. So it can be used to prevent use of mobile phones in examination halls, confidential rooms, etc. It is also useful for detecting the use of mobile phone for spying and unauthorized video transmission.

\section{Buffers and Drivers}

Buffers do not affect the logical state of a digital signal (i.e. a logic 1 input results in a logic 1 output whereas logic 0 input results in a logic 0 output). Buffers are normally used to provide extra current drive at the output but can also be used to regularize the logic present at an interface. Driver section is used to drive the relay where the output is complement of input which is applied to the drive but current will be amplified[6].

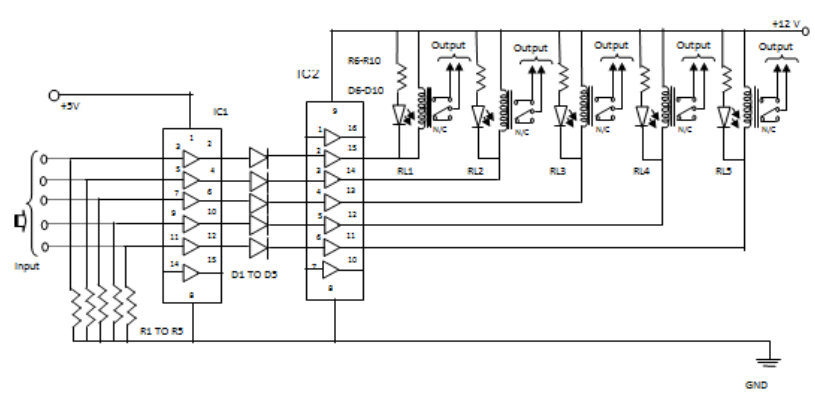

Figure.5 Buffer and Driver Circuit Diagram

\section{Circuit Description of Buffer and Driver}

The Hex Buffer/Inverter IC1's working voltage of $+5 \mathrm{~V}$ is applied at pin-1 and five control signals are applied at input pins 3, 5, 7, $9 \& 11$. Thus the signal supplying circuit [i.e. CONTROLLER] is isolated from this Buffer \& Driver circuit. Further the grounding resistors R1 to R5 prevents the abnormal voltage levels passing inside the IC1. The buffered outputs are acquired at pins $2,4,6,10, \& 12$. Thus the varying input is further stabilized and fed to signal diodes [D1 to D5]. As the load is inductive, there is a chance of producing back E.M.F. So to cope with this back E.M.F, signal diodes are used. But this signal level is not strong enough to drive the low impedance relay. So, IC2 Darlington driver is used. Its working voltage is $+12 \mathrm{~V}$ and only five input/output pins are used.

Relays

It is an electromagnetic device which is used to drive the load connected across the relay and the $\mathrm{o} / \mathrm{p}$ of relay can be connected to controller or load for further processing[5].

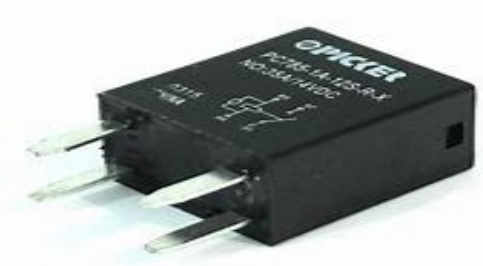

Figure.6 Relay Diagram 
LCD Display

LCD is a flat-panel display or other electronically modulated optical device that uses the light-modulating properties of liquid crystals. Liquid crystals do not emit light directly, instead using a backlight or reflector to produce images in color or monochrome. LCDs are available to display arbitrary images (as in a general-purpose computer display) or fixed images with low information content, which can be displayed or hidden, such as preset words, digits, and seven-segment displays, as in a digital clock. They use the same basic technology, except that arbitrary images are made up of a large number of small pixels, while other displays have larger elements. LCDs can either be normally on (positive) or off (negative), depending on the polarizer arrangement.

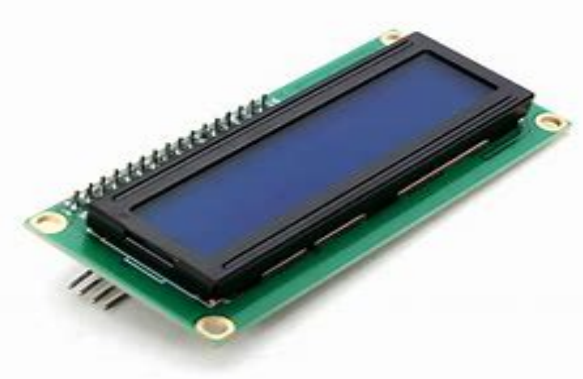

Figure.7 LCD Display

\section{Transmitter}

Transmitter or radio transmitter is an electronic device which produces radio waves with an antenna. The transmitter itself generates a radio frequency alternating current, which is applied to the antenna. When excited by this alternating current, the antenna radiates radio waves[8].

\section{Receiver}

Radio receiver also known as a receiver, wireless or simply radio is an electronic device that receives radio waves and converts the information carried by them to a usable form. It is used with an antenna. The antenna intercepts radio waves (electromagnetic waves) and converts them to tiny alternating currents which are applied to the receiver, and the receiver extracts the desired information[8].

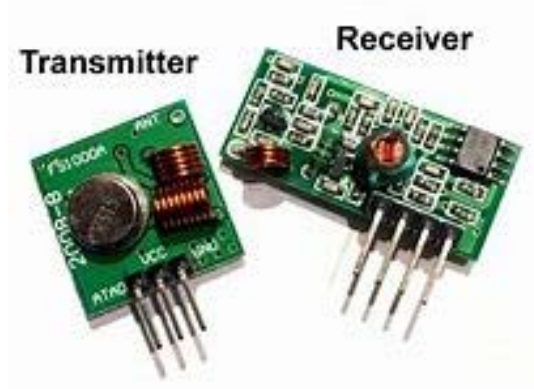

Figure.8 RF Transmitter and Receiver

\section{Buzzer}

A buzzer or beeper is an audio signaling device which may be mechanical, electromechanical, or piezoelectric (Piezo for short). Typical uses of buzzers and beepers include alarm devices, timers, and confirmation of user input such as a mouse click or keystroke[4].

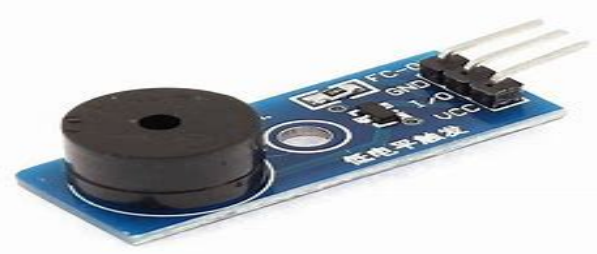

Figure.9 Buzzer

\section{III .Methodologies}

Drowsiness can become very dangerous, especially for such professions like drivers. Nowadays, more and more professions require long-term concentration. People, who work for transportation business (car and truck drivers, steersmen, airplane pilots), must keep a close eye on the road, so they can react to sudden events (e.g. Road accidents, animals on the road, etc.).And also alcohol consumption ,mobile usage while driving causes accident. So to avoid accidents caused due to these reasons we implement a system.

The aim of this project is to develop a prototype drowsiness detection system. The focus will be placed on designing a system that will accurately monitor the eye blink rate, alcohol consumption level and also mobile usage of the driver. In this project we use sensors to measure all these factors. This is a small system, so we can easily embed it on any vehicle. The Eye blink sensor is fixed to the driver. The eye blink sensor senses the 
movement of the eyeball. The sensor output is connected to a microcontroller.

The car engine starting system is directly controlled by the microcontroller. If the sensor detects the alcohol consumption from the sensor, it sends the signal to the microcontroller. The engine will be prevented from starting.

\section{CONCLUSION}

In this paper, we have the various methods available to determine the drowsiness state, usage of mobile while driving and alcohol detection of a driver. This paper also discusses the various ways in which drowsiness, mobile usage and alcohol detection can be detected in a simulated environment. The proposed system is used to avoid various road accidents caused by drowsy driving, using mobile while driving and alcohol consumption during driving.also this system used for security purpose of a driver to caution the driver if any of these state occurs.

This paper involves avoiding accident to unconsciousness through Eye blink. Here one eye blink sensor is fixed in vehicle where if driver lose his consciousness, then it alerts the driver through buzzer to prevent vehicle from accident. The alcohol are used for further safety system in the vehicle. Development of a hybrid microcontroller for a vehicle which also consists of an alcohol and temperature detector which will sense if the driver is drunk and would not start the vehicle. A complete study on road safety is going to be the next boom for the automobile industry for it to flourish and survive every human from the risk. The main advantage of this paper is the accuracy of using physiological parameters to detect drowsiness is really high. This helps in preventing most of the road accidents that occur due to fatigue.

\section{REFERENCES}

[1] Arun Sahayadhas,Kenneth Sundaraj, "Detecting Driver Drowsiness Based on Sensors A Review",pp.16937-16953, ISSN 1424-8220, Malaysia 2012

[2] Boon-Giin Lee and Wan-Young Chung, "Driver Alertness Monitoring Using Fusion of
Facial Features and Bio-Signals", IEEE Sensors journal, vol. 12, no.7,2012

[3] Dr.A.A.Gurjar, Neha A. Sarnaik,"Heart Attack Detection By Heartbeat Sensing using Internet Of Things : IoT'International Research Journal of Engineering and Technology (IRJET) e-ISSN: 2395-0056 Volume: 05 Issue: 03 | Mar-2018

[4] Bhavana T. Petkar, Bhagya C, Gagana T K, Lokesha K

"Automatic Driver Drowsiness Alert and Health Monitoring System using GSM"International Journal of Engineering Research \& Technology (IJERT) ISSN: 2278-0181

[5] Tigor Hamonangan Nasution, Muhammad Anggia Muchtar, Ikhsan Siregar, Ulfi Andayani, Esra Christian, Emerson Pascawira Sinulingga "Electrical Appliances Control Prototype by Using GSM Module and Arduino" $4^{\text {th }}$ International conference on Industrial Engineering and applications.

[6] Jamal Ahmad khan,Muhammad Shahzad"Sizing Buffers of IoT Edge Routers" $1^{\text {st }}$ International Conference June 2018 [7] Aarti Rao Jaladi, Karishma Khithani, Pankaja Pawar, Kiran Malvi, Gauri Sahoo,"Environmental Monitoring Using Wireless Sensor Networks(WSN) based on IOT"International Research Journal of Engineering and Technology (IRJET) e-ISSN: 2395 -0056 Volume: 04 Issue: 01 | Jan -2017

[8] R.A.Jain, Aditya Patil, Prasenjeet Nikam, Shubham More, Saurabh Totewar, "Women's safety using IOT" International Research Journal of Engineering and Technology (IRJET) e-ISSN: 2395 -0056 Volume: 04 Issue: 05 | May -2017 www.irjet.net p-ISSN: 23950072

[9] Parris, J., et.al, "Face and eye detection on hard datasets," Biometrics (IJCB), International Joint Conference on , vol., no., pp.l , 10 , 1-13 Oct. 2011

[10] X. Xie, R. Sudhakar and H. Zhuang, RealTime Eye Feature Tracking from a Video Image Sequence Using Kalman Filter,IIIEEE Transactions on Systems, Man, and Cybernetics, Vol. 25,No.12, pp. 1568-1577, 1995. 